\title{
Further Promotion of Cauchy Inequality
}

\author{
Fengqing Sun (Corresponding author) \\ Department of Computer Science, Dezhou Vocational and Technical College \\ Dezhou 253034, Shandong, China \\ E-mail: sunfengqing1963@126.com
}

\begin{abstract}
Cauchy inequality is a very important inequality in mathematics and physics, especially has wild application in resolving the proof of inequalities, which has significance for researching inequalities in mathematics. This paper promotes the original Cauchy inequality. The original $n$-dimension and 2-order Cauchy inequality is promoted to a $n$-dimension and $m$-order Cauchy inequality and the rigorous proof is also given. Simultaneously, this paper summarizes several corollaries for this $n$-dimension and $m$-order Cauchy inequality, and gives the corresponding proof.
\end{abstract}

Keywords: Cauchy inequality ( $n$-dimension and 2-order Cauchy inequality), Promotion of Cauchy inequality ( $n$-dimension and $m$-order Cauchy inequality), Mean inequality, Important corollary

\section{Cauchy inequality $(n$-dimension and 2-order Cauchy inequality)}

Suppose $a=\left(a_{1}, a_{2}, \cdots, a_{n}\right)$ and $b=\left(b_{1}, b_{2}, \cdots, b_{n}\right)$ are two real series, then

$$
\left(\sum_{i=1}^{n} a_{i} b_{i}\right)^{2} \leq\left(\sum_{i=1}^{n} a_{i}^{2}\right)\left(\sum_{i=1}^{n} b_{i}^{2}\right)
$$

and equal sign holds if and only if $a_{i}=0$ or $b_{i}=0$ or there exists constant $k$ satisfying $a_{i}=k b_{i}(i=1,2, \cdots, n)$.

2. Promotion of Cauchy inequality $(n$-dimension and $m$-order Cauchy inequality)

Suppose $a_{j}=\left(a_{j 1}, a_{j 2}, \cdots, a_{j n}\right)(j=1,2, \cdots, m)$ are $m$ real series(when $m$ is odd, $\left.a_{j i} \geq 0(j=1,2, \cdots, m ; i=1,2, \cdots, n)\right), m \geq$ 2 , then

$$
\left(\sum_{i=1}^{n} \prod_{j=1}^{m} a_{j i}\right)^{m} \leq \prod_{j=1}^{m}\left(\sum_{i=1}^{n} a_{j i}^{m}\right)
$$

and equal sign holds if and only if $a_{1 i}=0$ or $a_{2 i}=0 \cdots$ or $a_{m i}=0$ or existing m constants $k_{1}, k_{2}, \cdots, k_{m}$ satisfying $k_{1} a_{1 i}=k_{2} a_{2 i}=\cdots=k_{m} a_{m i}(i=1,2, \cdots, n)$.

Proof. It is divided into even and odd situations:

(1) When $m$ is even:

(1.1) When $a_{1 i}=0$ or $a_{2 i}=0 \cdots$ or $a_{m i}=0,(i=1,2, \cdots, n)$, the inequality holds obviously and the equal sign holds.

(1.2) When $\sum_{i=1}^{n} a_{j i}^{m} \neq 0,(j=1,2, \cdots, m)$, denoted $\sum_{i=1}^{n} a_{j i}^{m}=A_{j}(j=1,2, \cdots, m)$, then the original inequality turns into $\left(\sum_{i=1}^{n} \prod_{j=1}^{m} a_{j i}\right)^{m} \leq \prod_{j=1}^{m} A_{j}$, i.e. $\left|\sum_{i=1}^{n} \prod_{j=1}^{m} a_{j i}\right| \leq \sqrt[m]{\prod_{j=1}^{m} A_{j}}$. So we just prove this inequality: $\frac{\left|\sum_{i=1}^{n} \prod_{j=1}^{m} a_{j i}\right|}{\sqrt[m]{\prod_{j=1}^{m} A_{j}}} \leq 1$.

$$
\begin{aligned}
& \frac{\left|\sum_{i=1}^{n} \prod_{j=1}^{m} a_{j i}\right|}{\sqrt[m]{\prod_{j=1}^{m} A_{j}}} \leq \frac{\sum_{i=1}^{n}\left|\prod_{j=1}^{m} a_{j i}\right|}{\sqrt[m]{\prod_{j=1}^{m} A_{j}}}=\sum_{i=1}^{n} \frac{\left|\prod_{j=1}^{m} a_{j i}\right|}{\sqrt[m]{\prod_{j=1}^{m} A_{j}}}=\sum_{i=1}^{n} \sqrt[m]{\prod_{j=1}^{m} \frac{a_{j i}^{m}}{A_{j}}} \leq \sum_{i=1}^{n} \frac{\sum_{j=1}^{m} \frac{a_{j i}^{m}}{A_{j}}}{m}=\frac{1}{m} \sum_{j=1}^{m} \sum_{i=1}^{n} \frac{a_{j i}^{m}}{A_{j}} \\
= & \frac{1}{m} \sum_{j=1}^{m} \frac{\sum_{i=1}^{n} a_{j i}^{m}}{A_{j}}=\frac{1}{m} \sum_{j=1}^{m} \frac{A_{j}}{A_{j}}=1 .
\end{aligned}
$$


So $\left(\sum_{i=1}^{n} \prod_{j=1}^{m} a_{j i}\right)^{m} \leq \prod_{j=1}^{m}\left(\sum_{i=1}^{n} a_{j i}^{m}\right)$.

From the necessary and sufficient condition for the equal sign of absolute value inequality and mean inequality, we can see the equal sign holds if and only if $\left|\sum_{i=1}^{n} \prod_{j=1}^{m} a_{j i}\right|=\sum_{i=1}^{n}\left|\prod_{j=1}^{m} a_{j i}\right|$ and $\frac{a_{1 i}^{m}}{A_{1}}=\frac{a_{2 i}^{m}}{A_{2}}=\cdots=\frac{a_{m i}^{m}}{A_{m}}$, i.e. $k_{1} a_{1 i}=k_{2} a_{2 i}=\cdots=k_{m} a_{m i}(i=$ $1,2, \cdots, n)$, where $k_{j}= \pm \sqrt[m]{\frac{1}{A_{j}}}(j=1,2, \cdots, m)$.

(2) When $m$ is odd:

(2.1) When $a_{1 i}=0$ or $a_{2 i}=0 \cdots$ or $a_{m i}=0,(i=1,2, \cdots, n)$, the inequality holds obviously and the equal sign holds.

(2.2) When $\sum_{i=1}^{n} a_{j i}^{m} \neq 0$ (i.e. $\left.\sum_{i=1}^{n} a_{j i}^{m}>0\right),(j=1,2, \cdots, m)$, denoted $\sum_{i=1}^{n} a_{j i}^{m}=A_{j},(j=1,2, \cdots, m)$, then the original inequality turns into $\left(\sum_{i=1}^{n} \prod_{j=1}^{m} a_{j i}\right)^{m} \leq \prod_{j=1}^{m} A_{j}$, i.e. $\sum_{i=1}^{n} \prod_{j=1}^{m} a_{j i} \leq \sqrt[m]{\prod_{j=1}^{m} A_{j}}$. So we just prove this inequality: $\frac{\sum_{i=1}^{n} \prod_{j=1}^{m} a_{j i}}{\sqrt[m]{\prod_{j=1}^{m} A_{j}}} \leq 1$.

$$
\frac{\sum_{i=1}^{n} \prod_{j=1}^{m} a_{j i}}{\sqrt[m]{\prod_{j=1}^{m} A_{j}}}=\sum_{i=1}^{n} \frac{\prod_{j=1}^{m} a_{j i}}{\sqrt[m]{\prod_{j=1}^{m} A_{j}}}=\sum_{i=1}^{n} \sqrt[m]{\prod_{j=1}^{m} \frac{a_{j i}^{m}}{A_{j}}} \leq \sum_{i=1}^{n} \frac{\sum_{j=1}^{m} \frac{a_{j i}^{m}}{A_{j}}}{m}=\frac{1}{m} \sum_{j=1}^{m} \sum_{i=1}^{n} \frac{a_{j i}^{m}}{A_{j}}=\frac{1}{m} \sum_{j=1}^{m} \frac{\sum_{i=1}^{n} a_{j i}^{m}}{A_{j}}=\frac{1}{m} \sum_{j=1}^{m} \frac{A_{j}}{A_{j}}=1 .
$$

So $\left(\sum_{i=1}^{n} \prod_{j=1}^{m} a_{j i}\right)^{m} \leq \prod_{j=1}^{m}\left(\sum_{i=1}^{n} a_{j i}^{m}\right)$.

From the necessary and sufficient condition for the equal sign of mean inequality, we can see the equal sign holds if and only if $\frac{a_{1 i}^{m}}{A_{1}}=\frac{a_{2 i}^{m}}{A_{2}}=\cdots=\frac{a_{m i}^{m}}{A_{m}}$, i.e. $k_{1} a_{1 i}=k_{2} a_{2 i}=\cdots=k_{m} a_{m i},(i=1,2, \cdots, n)$, where $k_{j}=\sqrt[m]{\frac{1}{A_{j}}},(j=1,2, \cdots, m)$.

From the above (1) and (2), the inequality $\left(\sum_{i=1}^{n} \prod_{j=1}^{m} a_{j i}\right)^{m} \leq \prod_{j=1}^{m}\left(\sum_{i=1}^{n} a_{j i}^{m}\right)$ holds.

\section{Several important corollaries for $n$-dimension and $m$-order Cauchy inequality( Only simple proof is given here)}

Corollary 1. Suppose $a_{1}, a_{2}, \cdots, a_{n}$ are real numbers(when $m$ is odd, $a_{i} \geq 0(i=1,2, \cdots, n)$ ), then

$$
\left(\sum_{i=1}^{n} a_{i}\right)^{m} \leq n^{m-1}\left(\sum_{i=1}^{n} a_{i}^{m}\right)
$$

and equal sign holds if and only if $a_{1}=a_{2}=\cdots=a_{n}$, where $m \geq 2$ is a positive integer.

Proof: For the $n$-dimension and $m$-order Cauchy inequality $\left(\sum_{i=1}^{n} \prod_{j=1}^{m} a_{j i}\right)^{m} \leq \prod_{j=1}^{m}\left(\sum_{i=1}^{n} a_{j i}^{m}\right)$, let $a_{1 i}=a_{2 i}=\cdots=a_{m-1, i}=$ $1, a_{m i}=a_{i},(i=1,2, \cdots, n)$, we obtain $\left(\sum_{i=1}^{n} a_{i}\right)^{m} \leq n^{m-1}\left(\sum_{i=1}^{n} a_{i}^{m}\right)$.

And from the sufficient and necessary condition for the equal sign of the above $n$-dimension and $m$-order Cauchy inequality, we have the equal sign of this inequality holds if and only if $a_{1}=a_{2}=\cdots=a_{n}$.

Corollary 2. Suppose $a_{j}=\left(a_{j 1}, a_{j 2}, \cdots, a_{j n}\right)(j=1,2, \cdots, m)$ are $m$ non-negative real series $(m \geq 2)$, then

$$
\prod_{j=1}^{m}\left(\sum_{i=1}^{n} a_{j i}\right) \geq\left(\sum_{i=1}^{n} \sqrt[m]{\left.\prod_{j=1}^{m} a_{j i}\right)^{m}}\right.
$$

and equal sign holds if and only if $a_{1 i}=0$ or $a_{2 i}=0 \cdots$ or $a_{m i}=0$ or there exist positive constants $k_{1}, k_{2}, \cdots, k_{m}$ satisfying $k_{1} a_{1 i}=k_{2} a_{2 i}=\cdots=k_{m} a_{m i}(i=1,2, \cdots, n)$.

Proof: From the above $n$-dimension and $m$-order Cauchy inequality, we have

$$
\left(\sum_{i=1}^{n} \sqrt[m]{\left.\prod_{j=1}^{m} a_{j i}\right)^{m}}=\left(\sum_{i=1}^{n} \prod_{j=1}^{m} \sqrt[m]{a_{j i}}\right)^{m} \leq \prod_{j=1}^{m}\left[\sum_{i=1}^{n}\left(\sqrt[m]{a_{j i}}\right)^{m}\right]=\prod_{j=1}^{m}\left(\sum_{i=1}^{n} a_{j i}\right) .\right.
$$

i.e.

$$
\prod_{j=1}^{m}\left(\sum_{i=1}^{n} a_{j i}\right) \geq\left(\sum_{i=1}^{n} \sqrt[m]{\left.\prod_{j=1}^{m} a_{j i}\right)^{m}}\right.
$$


and equal sign holds if and only if $a_{1 i}=0$ or $a_{2 i}=0 \cdots$ or $a_{m i}=0$ or there exist positive constants $k_{1}, k_{2}, \cdots, k_{m}$ satisfying $k_{1} a_{1 i}=k_{2} a_{2 i}=\cdots=k_{m} a_{m i}(i=1,2, \cdots, n)$.

Corollary 3. Suppose $a_{1}, a_{2}, \cdots, a_{n}$ are real numbers(when $m$ is odd, $a_{i} \geq 0(i=1,2, \cdots, n)$ ) and $p_{1}, p_{2}, \cdots p_{n}$ are $n$ positive numbers, then

$$
\sum_{i=1}^{n} p_{i} a_{i}^{m} \geq \frac{\left(\sum_{i=1}^{n} a_{i}\right)^{m}}{\left(\sum_{i=1}^{n} \frac{1}{\sqrt[m-1]{p_{i}}}\right)^{m-1}} .
$$

and equal sign holds if and only if $\sqrt[m-1]{p_{1}} a_{1}=\sqrt[m-1]{p_{2}} a_{2}=\cdots=\sqrt[m-1]{p_{n}} a_{n}$, where $m \geq 2$ is a positive integer.

Proof: For the $n$-dimension and $m$-order Cauchy inequality $\left(\sum_{i=1}^{n} \prod_{j=1}^{m} a_{j i}\right)^{m} \leq \prod_{j=1}^{m}\left(\sum_{i=1}^{n} a_{j i}^{m}\right)$, let $a_{1 i}=\sqrt[m n]{p_{i}} a_{i}, a_{2 i}=a_{3 i}=\cdots=$ $a_{m i}=\frac{1}{\sqrt[m(m-1)]{p_{i}}},(i=1,2, \cdots, n)$, we obtain

$$
\left(\sum_{i=1}^{n} a_{i}\right)^{m} \leq \sum_{i=1}^{n}\left(\sqrt[n]{p_{i}} a_{i}\right)^{m}\left(\sum_{i=1}^{n} \frac{1}{\sqrt[m-1]{p_{i}}}\right)^{m-1}=\sum_{i=1}^{n} p_{i} a_{i}^{m}\left(\sum_{i=1}^{n} \frac{1}{\sqrt[m-1]{p_{i}}}\right)^{m-1},
$$

i.e.

$$
\sum_{i=1}^{n} p_{i} a_{i}^{m} \geq \frac{\left(\sum_{i=1}^{n} a_{i}\right)^{m}}{\left(\sum_{i=1}^{n} \frac{1}{\sqrt[m-1]{p_{i}}}\right)^{m-1}} .
$$

And from the sufficient and necessary condition for the equal sign of the above $n$-dimension and $m$-order Cauchy inequality, we have the equal sign of this inequality holds if and only if $\sqrt[m-1]{p_{1}} a_{1}=\sqrt[m-1]{p_{2}} a_{2}=\cdots=\sqrt[m-1]{p_{n}} a_{n}$.

Corollary 4. Suppose $a_{1}, a_{2}, \cdots, a_{n}$ are non-negative real numbers and $p_{1}, p_{2}, \cdots p_{n}$ are $n$ nonzero real numbers(when $m$ is odd, $\left.p_{i}>0(i=1,2, \cdots, n)\right)$, then

$$
\left|\sum_{i=1}^{n} p_{i} \sqrt[m]{a_{i}}\right| \leq\left(\sum_{i=1}^{n} \sqrt[m-1]{p_{i}^{m}}\right)^{\frac{m-1}{m}} \sqrt[m]{\sum_{i=1}^{n} a_{i}}
$$

and equal sign holds if and only if $\frac{a_{1}}{\sqrt[m-1]{p_{1}}}=\frac{a_{2}}{\sqrt[m-1]{p_{2}}}=\cdots=\frac{a_{n}}{\sqrt[m-1]{p_{n}}}$, where $m \geq 2$ is a positive integer.

Proof: For the $n$-dimension and $m$-order Cauchy inequality $\left(\sum_{i=1}^{n} \prod_{j=1}^{m} a_{j i}\right)^{m} \leq \prod_{j=1}^{m}\left(\sum_{i=1}^{n} a_{j i}^{m}\right)$, let $a_{1 i}=a_{2 i}=\cdots=a_{m-1, i}=$ $\sqrt[m-1]{p_{i}}, a_{m i}=\sqrt[m]{a_{i}}(i=1,2, \cdots, n)$, we obtain

$$
\left(\sum_{i=1}^{n} p_{i} \sqrt[m]{a_{i}}\right)^{m} \leq\left(\sum_{i=1}^{n} \sqrt[m-1]{p_{i}^{m}}\right)^{m-1}\left[\sum_{i=1}^{n}\left(\sqrt[m]{a_{i}}\right)^{m}\right]=\left(\sum_{i=1}^{n} \sqrt[m-1]{p_{i}^{m}}\right)^{m-1}\left(\sum_{i=1}^{n} a_{i}\right) .
$$

So

$$
\left|\sum_{i=1}^{n} p_{i} \sqrt[m]{a_{i}}\right| \leq\left(\sum_{i=1}^{n} \sqrt[m-1]{p_{i}^{m}}\right)^{\frac{m-1}{m}} \sqrt[m]{\sum_{i=1}^{n} a_{i}}
$$

And from the sufficient and necessary condition for the equal sign of the above $n$-dimension and $m$-order Cauchy inequality, we have the equal sign of this inequality holds if and only if $\frac{a_{1}}{\sqrt[m-1]{p_{1}}}=\frac{a_{2}}{\sqrt[m-1]{p_{2}}}=\cdots=\frac{a_{n}}{\sqrt[m-1]{p_{n}}}$.

Corollary 5. Suppose $a_{j}=\left(a_{j 1}, a_{j 2}, \cdots, a_{j n}\right)(j=1,2, \cdots, m)$ are $m$ real $\operatorname{series}(m \geq 2)$ and $\prod_{j=1}^{m} a_{j i}>0, a_{1 i} a_{j i}>0(j=$ $2,3, \cdots, m),(i=1,2, \cdots, n)$, then

$$
\prod_{j=2}^{m} \sum_{i=1}^{n} \frac{a_{1 i}}{a_{j i}} \geq \frac{\left(\sum_{i=1}^{n} a_{1 i}\right)^{m}}{\sum_{i=1}^{n} \prod_{j=1}^{m} a_{j i}},
$$

and equal sign holds if and only if $a_{21}=a_{22}=\cdots=a_{2 n}, a_{31}=a_{32}=\cdots=a_{3 n}, \cdots$, and $a_{m 1}=a_{m 2}=\cdots=a_{m n}$.

Proof: Replace $a_{1 i}$ by $\sqrt[m]{\prod_{j=1}^{m} a_{j i}}$ and $a_{j i}$ by $\sqrt[m]{\frac{a_{1 i}}{a_{j i}}},(j=2,3, \cdots, m),(i=1,2, \cdots, n)$ in the above $n$-dimension and $m$-order Cauchy inequality $\left(\sum_{i=1}^{n} \prod_{j=1}^{m} a_{j i}\right)^{m} \leq \prod_{j=1}^{m}\left(\sum_{i=1}^{n} a_{j i}^{m}\right)$, we obtain

$$
\left(\sum_{i=1}^{n} \sqrt[m]{\prod_{j=1}^{m} a_{j i}} \cdot \prod_{j=2}^{m} \sqrt[m]{\frac{a_{1 i}}{a_{j i}}}\right)^{m} \leq \sum_{i=1}^{n}\left(\sqrt[m]{\prod_{j=1}^{m} a_{j i}}\right)^{m} \cdot \prod_{j=2}^{m}\left[\sum_{i=1}^{n}\left(\sqrt[m]{\frac{a_{1 i}}{a_{j i}}}\right)^{m}\right]
$$


i.e. $\left(\sum_{i=1}^{n} a_{1 i}\right)^{m} \leq \sum_{i=1}^{n} \prod_{j=1}^{m} a_{j i} \cdot \prod_{j=2}^{m}\left(\sum_{i=1}^{n} \frac{a_{1 i}}{a_{j i}}\right)$.

So

$$
\prod_{j=2}^{m} \sum_{i=1}^{n} \frac{a_{1 i}}{a_{j i}} \geq \frac{\left(\sum_{i=1}^{n} a_{1 i}\right)^{m}}{\sum_{i=1}^{n} \prod_{j=1}^{m} a_{j i}},
$$

And from the sufficient and necessary condition for the equal sign of the above $n$-dimension and $m$-order Cauchy inequality, we have the equal sign of this inequality holds if and only if $a_{21}=a_{22}=\cdots=a_{2 n}, a_{31}=a_{32}=\cdots=a_{3 n}, \cdots$, and $a_{m 1}=a_{m 2}=\cdots=a_{m n}$.

\section{References}

Cheng, Legen. (2004). Proof and application of Cauchy inequality. Journal of West Anhui University, 2.

Chen, Xihua. (2005). Eight proof of 2-dimension Cauchy inequality. Journal of Hebei Polytechnic University , 2.

Li, Shenghong. Mean inequality and Cauchy inequality(small series of mathematical Olympiad), Shanghai: East China Normal University Press, (Chapter 4).

Luo, Kui \& Wang, Ping. (2002). Improvement of Cauchy inequality. Journal of Jingzhou Normal College , 2.

Wang, Lianhua, Li, Ye \& Li, Zhanguo. (2003). Proof and application of Cauchy inequality. Journal of Henan Institute of Education(Natural Science Edition), 2.

Wang, Xiaofeng. (2008). Discussion of Cauchy inequality. Social Science/Culture, Science, Education, Sports, 11-17.

Yuan, Gaowen. (2002). Cauchy inequality in Euclidean space. Journal of Further Education of Shanxi Normal University, 3.

Zong, Xufeng. (2000). Promotion of Cauchy inequality. Journal of Qufu Normal University (Natural Science Edition), 4. 